\title{
Publisher's Note: Revisiting universality of the liquid-gas critical point in two dimensions [Phys. Rev. E 96, 062124 (2017)]
}

Max Yarmolinsky and Anatoly Kuklov

(Q) (Received 18 October 2018; published 29 October 2018)

DOI: 10.1103/PhysRevE.98.049901

This paper was published online on 15 December 2017 with an error in the caption to Fig. 11. The second sentence in the caption of Fig. 11 should read as "The value of the exponent $\mu=0.532 \pm 0.005$ is consistent with the Onsager value $\mu=8 / 15$." The caption has been corrected as of 22 October 2018. The caption is incorrect in the printed version of the journal. 